\title{
Maximum principles and overdetermined problems for Hessian equations
}

\section{Principes du maximum et problèmes surdéterminés des équations Hessiennes}

\author{
Cristian Enache ${ }^{1}$, Monica Marras ${ }^{2}$ and Giovanni Porru² \\ ${ }^{1}$ Department of Mathematics and Statistics, American University of Sharjah, Sharjah, 26666, UAE \\ cenache@aus.edu \\ ${ }^{2}$ Dipartimento di Matematica e Informatica, University of Cagliari, Cagliari, Italy \\ mmarras@unica.it,porru@unica.it
}

\begin{abstract}
In this article we investigate some Hessian type equations. Our main aim is to derive new maximum principles for some suitable P-functions, in the sense of L.E. Payne, that is for some appropriate functional combinations of $u(x)$ and its derivatives, where $u(x)$ is a solution of the given Hessian type equations. To find the most suitable P-functions, we first investigate the special case of a ball, where the solution of our Hessian equations is radial, since this case gives good hints on the best functional to be considered later, for general domains. Next, we construct some elliptic inequalities for the well-chosen P-functions and make use of the classical maximum principles to get our new maximum principles. Finally, we consider some overdetermined problems and show that they have solutions when the underlying domain has a certain shape (spherical or ellipsoidal).

2010 Mathematics Subject Classification: 35B50; 35B45; 35J25; 35J60; 35J96

KEYWORDS. Monge-Ampère equations, Hessian Equations, maximum principles, overdetermined problems.
\end{abstract}

\section{Introduction}

One of the most important and useful tools used in the study of partial differential equations is the maximum principle, which is nothing else that a natural extension to higher dimensions of the following elementary fact from calculus courses: any real-valued function $f$, which satisfies the inequality $f^{\prime \prime}>0$ on an open interval $(a, b)$, cannot attain its maximum at a point of $(a, b)$ (for more details, we refer the reader to the fundamental book of M.H. Protter and H.F. Weinberger [14]). For instance, if we consider a bounded domain $\Omega \subset \mathbb{R}^{n}, n \geq 2$, and a function $u(x)$ satisfying the equation

$$
\Delta u=1 \text { in } \Omega \text {, }
$$

then the maximum principle guarantees that $u(x)$ cannot attain its maximum in $\Omega$.

In this paper we do not plan to investigate this kind of classical maximum principles, but rather maximum principles for P-functions, in the sense of L.E. Payne. More precisely, we will develop new maximum principles for some appropriate functional combinations of $u(x)$ and its derivatives (see the book of R. Sperb [18]), where $u(x)$ will be the solution of some Hessian equations. Moreover, we will find the best possible candidates for such maximum principles, in order to get the so-called best maximum principles. These candidates are the functional combinations which are identically constant on some particular type of domains, such as, for instance, domains with spherical or ellipsoidal shape. For instance, if $\Omega$ is the unit ball centered at the origin, then the function

$$
u(x)=\frac{|x|^{2}-1}{2 n},
$$


satisfies $\Delta u=1$ in $\Omega$. Moreover, one can easily notice that the auxiliary functional defined as

$$
\phi(x):=|\nabla u|^{2}-\frac{2}{n} u,
$$

is identically constant on $\Omega$. Therefore, a question of interest is to investigate what is happening with the P-function $\phi$ when $\Omega$ is not a ball, but any other general domain. In such a case, for a general bounded domain $\Omega$, C. Miranda proved that $\phi(x)$ satisfies a maximum principles, in the sense that $\phi(x)$ attains its maximum value on $\partial \Omega$, the boundary of $\Omega$ (see R. Sperb [18]).

Such maximum principles for appropriate P-functions have several important applications. First of all, they are very useful in getting isoperimetric estimates. For instance, when $u(x)$ also satisfies a Dirichlet boundary condition, that is $u=0$ on $\partial \Omega$, and $\Omega$ is a strictly convex domain, then one can use the above mentioned Miranda's maximum principle and some computations in normal coordinates with respect to $\partial \Omega$, to obtain the following estimates (see R. Sperb, Ch. 6 [18]):

$$
|\nabla u| \leq \frac{1}{n H_{\min }}, \quad u(x) \geq-\frac{1}{2 n H_{\min }^{2}},
$$

where $H_{\min }$ is the minimum value of the mean curvature of $\partial \Omega$. These estimates are isoperimetric, since the equality holds if and only if $\Omega$ is a ball. The sufficient condition can be proved by using a second interesting application of the maximum principle for $\phi(x)$. Obviously, if we have equality above, then $u(x)$ satisfies the following overdetermined problem

$$
\left\{\begin{array}{l}
\Delta u=1 \text { in } \Omega, \\
u=0 \text { on } \partial \Omega, \\
|\nabla u|=c=\text { const. on } \partial \Omega .
\end{array}\right.
$$

It was proved by J. Serrin, in a seminal paper [17], that this problem has a solution if and only if $\Omega$ is a ball. His method of proof is based on the classical maximum principle and the moving plane method. In a subsequent paper [19], H.F. Weinberger proved the same result making use of the maximum principle for $\phi(x)$, mentioned above. More precisely, using the boundary conditions on $u(x)$ and $|\nabla u(x)|$, the maximum principle implies that

$$
\text { either }(I) \phi(x)<c \text { in } \Omega, \quad \text { or }(I I) \phi(x) \equiv c \text { on } \Omega \text {. }
$$

Next, using a Pohozaev type identity, $(I)$ can be ruled out, so from $(I I)$ we get that

$$
\Delta \phi(x)=0 \text { in } \Omega
$$

which implies that $D^{2} u=\frac{1}{n} I_{n}$, so $u$ is radial and $\Omega$ is a ball.

Finally, let us mention that the above maximum principle for $\phi$ and its applications have been extended by many authors to more general classes of nonlinear equations in divergence form (see [13], [18] and the references therein). The aim of this paper is to bring some new contributions, by extending such results to some fully nonlinear equations of Hessian type.

The paper is organized as follows. In Section 2, we first analyze the case of the ball, which gives inspiring hints about the P-functions to be considered. Thereafter, we develop some maximum principles for such P-functions. In Section 3, we consider some overdetermined problems and find the shapes of the underlying domain $\Omega$ for which we have solutions. 
Throughout this paper the summation convention over repeated indices is employed and the following notations are adopted: $u_{i}=\partial u / \partial x_{i}, u_{i j}=\partial^{2} u /\left(\partial x_{i} \partial x_{j}\right)$. Using these notations we have, for instance,

$$
u_{i j} u_{i} u_{j}=\sum_{i=1}^{n} \sum_{j=1}^{n} \frac{\partial^{2} u}{\partial x_{i} \partial x_{j}} \frac{\partial u}{\partial x_{i}} \frac{\partial u}{\partial x_{j}}
$$

\section{Maximum principles}

Let $\Omega$ be a bounded domain in $\mathbb{R}^{n}, n \geq 2$, and let $\kappa_{1}, \cdots, \kappa_{n-1}$ be the principal curvatures of the boundary $\partial \Omega$ at a point $x \in \partial \Omega$. The $\kappa$-th curvature of $\partial \Omega$ at the point $x$ is defined as

$$
H_{(\kappa)}:=H_{(\kappa)}\left(\kappa_{1}, \cdots, \kappa_{n-1}\right)
$$

where $H_{(\kappa)}\left(\kappa_{1}, \cdots, \kappa_{n-1}\right)$ denotes the $\kappa$-th elementary symmetric function of the curvatures $\kappa_{1}, \cdots, \kappa_{n-1}$. We say that $\Omega$ is $\kappa$-convex if $H_{(i)}\left(\kappa_{1}, \cdots, \kappa_{n-1}\right) \geq 0$ for $i=1, \cdots, \kappa$ and for every $x \in \partial \Omega$. In this paper we assume $\Omega$ to be $\kappa$-convex.

Let us recall the definition of $\kappa$-convex functions in $\Omega$. If $u=u(x)$ is a $C^{2}$ function, let $\lambda_{1}, \cdots, \lambda_{n}$ be the eigenvalues of the Hessian matrix $D^{2} u$ and $\sigma_{(\kappa)}$ be the $\kappa$-th elementary symmetric function of the eigenvalues $\lambda_{1}, \cdots, \lambda_{n}$. Then we say that $u$ is $\kappa$-convex if $\sigma_{(i)} \geq 0$ for $i=1, \cdots, \kappa$ and for every $x \in \Omega$.

Let us now consider the following Hessian problem:

$$
\sigma_{(\kappa)}\left(D^{2} u\right)=f(u) \text { in } \Omega, u=0 \text { on } \partial \Omega,
$$

where $f$ is a positive smooth function. We have $\sigma_{(1)}=\Delta u$ and $\sigma_{(n)}=\operatorname{det}\left(D^{2} u\right)$. We say that a solution $u$ of (1) is admissible if it is $\kappa$-convex. We refer to [16] for a discussion on existence and uniqueness of Problem (1).

Next, let us recall the definition of the $\nu$-th Newton tensor associated with the Hessian matrix $D^{2} u$ :

$$
T_{(\nu)}:=\sigma_{(\nu)} I-T_{(\nu-1)} D^{2} u, \quad \nu=1, \cdots, n, \quad T_{(0)}:=I,
$$

where $I$ is the identity matrix. An useful equation is the following (see [15])

$$
\frac{\partial \sigma_{(\nu)}}{\partial u_{i j}}=T_{(\nu-1)}^{i j}, \quad i, j=1, \cdots, n .
$$

Since $D^{2} u$ is symmetric, also the matrices $T_{(\nu)}$ are symmetric.

For $1 \leq \nu \leq \kappa$, we consider the P-function

$$
\phi(x):=T_{(\nu-1)}^{k l} u_{k} u_{l}-2\left(\begin{array}{c}
n-1 \\
\nu-1
\end{array}\right)\left(\begin{array}{l}
n \\
\kappa
\end{array}\right)^{-\frac{\nu}{\kappa}} \int_{0}^{u} f^{\frac{\nu}{\kappa}}(t) d t
$$

as well as the P-function

$$
\psi(x):=T_{(\nu-1)}^{k l} u_{k} u_{l}-2\left(\begin{array}{c}
n-1 \\
\nu-1
\end{array}\right)\left(\begin{array}{l}
n \\
\kappa
\end{array}\right)^{-\frac{\nu}{\kappa}} c_{0}^{\frac{\nu}{\kappa}} u
$$

where $c_{0}=f\left(m_{0}\right), m_{0}=\min _{\Omega} u(x)$. We note that when $f$ is a constant, $\phi(x)=\psi(x)$.

In this section we investigate the following problem: Do these P-functions, $\phi$ and $\psi$, attain their maximum or minimum values at the boundary $\partial \Omega$ ? 


\subsection{The radial case}

In this subsection we'll investigate the case of a ball and obtain the following result:

Theorem 2.1. Let $\Omega$ be a ball, $u$ be a (radial) solution of (1), $\phi$ be the P-functions defined in (2) and $\psi$ be the P-function defined in (3) We have:

(i) if $f(t)$ is non-decreasing, then $\phi^{\prime}(r) \geq 0$;

(ii) if $f(t)$ is non-increasing, then $\phi^{\prime}(r) \leq 0$;

(iii) if $f(t)$ is non-decreasing, then $\psi^{\prime}(r) \geq 0$.

Proof. We note that our equation (1) as well as our P-functions (2) and (3) are invariant under a rigid rotation. Therefore, we can assume $x=(r, 0, \cdots, 0)$ and we thus have

$$
u_{1}=u^{\prime}, \quad u_{i}=0 \quad i=2, \cdots, n
$$

respectively

$$
D^{2} u=\operatorname{diag}\left\{u^{\prime \prime}, \frac{u^{\prime}}{r}, \cdots, \frac{u^{\prime}}{r}\right\}
$$

Let us consider first the P-function $\phi(x)$, defined in (2), which can be written now as

$$
\phi(r)=T_{(\nu-1)}^{11}\left(u^{\prime}\right)^{2}-\alpha \int_{0}^{u} f(t)^{\frac{\nu}{\kappa}} d t
$$

where

$$
\alpha:=2\left(\begin{array}{l}
n-1 \\
\nu-1
\end{array}\right)\left(\begin{array}{l}
n \\
\kappa
\end{array}\right)^{-\frac{\nu}{\kappa}}
$$

. We claim that

$$
T_{(\nu)}^{11}=\left(\begin{array}{c}
n-1 \\
\nu
\end{array}\right)\left(\frac{u^{\prime}}{r}\right)^{\nu}, \quad \nu=0, \cdots, n-1 .
$$

Indeed, the relation is true (trivially) for $\nu=0$. Next, we proceed by induction, assuming that (5) holds for $\nu-1$, that is,

$$
T_{(\nu-1)}^{11}=\left(\begin{array}{l}
n-1 \\
\nu-1
\end{array}\right)\left(\frac{u^{\prime}}{r}\right)^{\nu-1}
$$

Since

$$
T_{(\nu)}=\sigma_{(\nu)} I-T_{(\nu-1)} D^{2} u,
$$

by (4) we find

$$
T_{(\nu)}^{11}=\left(\begin{array}{c}
n-1 \\
\nu-1
\end{array}\right) u^{\prime \prime}\left(\frac{u^{\prime}}{r}\right)^{\nu-1}+\left(\begin{array}{c}
n-1 \\
\nu
\end{array}\right)\left(\frac{u^{\prime}}{r}\right)^{\nu}-\left(\begin{array}{c}
n-1 \\
\nu-1
\end{array}\right) u^{\prime \prime}\left(\frac{u^{\prime}}{r}\right)^{\nu-1} .
$$

After simplification we find (5), and the claim is proved.

So, we can write

$$
\phi(r)=\left(\begin{array}{l}
n-1 \\
\nu-1
\end{array}\right) \frac{\left(u^{\prime}\right)^{\nu+1}}{r^{\nu-1}}-\alpha \int_{0}^{u} f^{\frac{\nu}{\kappa}}(t) d t
$$


and obtain

$$
\phi^{\prime}(r)=u^{\prime}\left\{\left(\begin{array}{l}
n-1 \\
\nu-1
\end{array}\right)\left[(\nu+1)\left(\frac{u^{\prime}}{r}\right)^{\nu-1} u^{\prime \prime}-(\nu-1)\left(\frac{u^{\prime}}{r}\right)^{\nu}\right]-\alpha f^{\frac{\nu}{\kappa}}\right\} .
$$

Equation (1) in the radial case reads as

$$
\left(\begin{array}{c}
n-1 \\
\kappa-1
\end{array}\right)\left(\frac{u^{\prime}}{r}\right)^{\kappa-1} u^{\prime \prime}+\left(\begin{array}{c}
n-1 \\
\kappa
\end{array}\right)\left(\frac{u^{\prime}}{r}\right)^{\kappa}=f,
$$

from which we find

$$
\left(\frac{u^{\prime}}{r}\right)^{\kappa-1} u^{\prime \prime}=\left(\begin{array}{c}
n-1 \\
\kappa-1
\end{array}\right)^{-1}\left(f-\left(\begin{array}{c}
n-1 \\
\kappa
\end{array}\right)\left(\frac{u^{\prime}}{r}\right)^{\kappa}\right) .
$$

Insertion of the latter equation into (6) yields

$$
\begin{aligned}
\phi^{\prime}(r) & =u^{\prime}\left\{( \begin{array} { c } 
{ n - 1 } \\
{ \nu - 1 }
\end{array} ) \left[( \nu + 1 ) ( \begin{array} { c } 
{ n - 1 } \\
{ \kappa - 1 }
\end{array} ) ^ { - 1 } \left(\left(\frac{u^{\prime}}{r}\right)^{\nu-\kappa} f\right.\right.\right. \\
& \left.\left.\left.-\left(\begin{array}{c}
n-1 \\
\kappa
\end{array}\right)\left(\frac{u^{\prime}}{r}\right)^{\nu}\right)-(\nu-1)\left(\frac{u^{\prime}}{r}\right)^{\nu}\right]-\alpha f^{\frac{\nu}{\kappa}}\right\} .
\end{aligned}
$$

By (7) we have

$$
\begin{aligned}
& k\left(\frac{u^{\prime}}{r}\right)^{\kappa-1} u^{\prime \prime}+(n-\kappa)\left(\frac{u^{\prime}}{r}\right)^{\kappa}=k\left(\begin{array}{c}
n-1 \\
\kappa-1
\end{array}\right)^{-1} f \\
& \left(\left(u^{\prime}\right)^{\kappa}\right)^{\prime}+\frac{n-\kappa}{r}\left(u^{\prime}\right)^{\kappa}=r^{\kappa-1} k\left(\begin{array}{c}
n-1 \\
\kappa-1
\end{array}\right)^{-1} f,
\end{aligned}
$$

and

$$
\left(r^{n-\kappa}\left(u^{\prime}\right)^{\kappa}\right)^{\prime}=r^{n-1} k\left(\begin{array}{c}
n-1 \\
\kappa-1
\end{array}\right)^{-1} f .
$$

Since $u^{\prime}(0)=0$, from (9) we find that $u^{\prime}(r)>0$. If $f(t)$ is non-decreasing then $f(u(r))$ if nondecreasing. Therefore, integrating (9) we find

$$
r^{n-\kappa}\left(u^{\prime}\right)^{\kappa} \leq \frac{r^{n}}{n} k\left(\begin{array}{c}
n-1 \\
\kappa-1
\end{array}\right)^{-1} f
$$

and

$$
\left(\frac{u^{\prime}}{r}\right)^{\kappa} \leq\left(\begin{array}{l}
n \\
\kappa
\end{array}\right)^{-1} f
$$

Insertion of the latter estimate into (8) yields

$$
\begin{aligned}
\phi^{\prime}(r) & \geq u^{\prime}\left\{( \begin{array} { l } 
{ n - 1 } \\
{ \nu - 1 }
\end{array} ) \left[( \nu + 1 ) ( \begin{array} { l } 
{ n - 1 } \\
{ \kappa - 1 }
\end{array} ) ^ { - 1 } \left[\left(\begin{array}{l}
n \\
\kappa
\end{array}\right)\right.\right.\right. \\
& \left.\left.\left.-\left(\begin{array}{c}
n-1 \\
\kappa
\end{array}\right)\right]-(\nu-1)\right]\left(\begin{array}{l}
n \\
\kappa
\end{array}\right)^{-\frac{\nu}{\kappa}}-\alpha\right\} f^{\frac{\nu}{\kappa}} .
\end{aligned}
$$

Since

$$
\left(\begin{array}{l}
n-1 \\
\kappa-1
\end{array}\right)^{-1}\left[\left(\begin{array}{l}
n \\
\kappa
\end{array}\right)-\left(\begin{array}{c}
n-1 \\
\kappa
\end{array}\right)\right]=1
$$


recalling the value of $\alpha$ we find

$$
\phi^{\prime}(r) \geq u^{\prime}\left\{2\left(\begin{array}{l}
n-1 \\
\nu-1
\end{array}\right)\left(\begin{array}{l}
n \\
\kappa
\end{array}\right)^{-\frac{\nu}{\kappa}}-\alpha\right\} f^{\frac{\nu}{\kappa}}=0 .
$$

Part (i) of the theorem is proved.

If $f(t)$ is non-increasing then $f(u(r))$ if non-increasing. Integrating (9) now we find

$$
\left(\frac{u^{\prime}}{r}\right)^{\kappa} \geq\left(\begin{array}{l}
n \\
\kappa
\end{array}\right)^{-1} f
$$

Insertion of the latter estimate into (8) yields

$$
\phi^{\prime}(r) \leq u^{\prime}\left\{2\left(\begin{array}{l}
n-1 \\
\nu-1
\end{array}\right)\left(\begin{array}{l}
n \\
\kappa
\end{array}\right)^{-\frac{\nu}{\kappa}}-\alpha\right\} f^{\frac{\nu}{\kappa}}=0 .
$$

Part (ii) of the theorem is proved.

The proof of the result for $\psi$ is similar. If $f(t)$ is non-decreasing, in place of (11) we find

$$
\psi^{\prime}(r) \geq u^{\prime} 2\left(\begin{array}{l}
n-1 \\
\nu-1
\end{array}\right)\left(\begin{array}{l}
n \\
\kappa
\end{array}\right)^{-\frac{\nu}{\kappa}}\left\{f^{\frac{\nu}{\kappa}}-c_{0}^{\frac{\nu}{\kappa}}\right\} \geq 0
$$

and the part (iii) of the theorem is also proved.

Remark. If $f(t)$ is non-increasing, then one can find $\psi^{\prime}(r) \leq 0$, provided that the constant $c_{0}=f\left(m_{0}\right)$ is replaced by $c_{1}=f(0)$. In case $f(t)$ is a constant, Theorem 2.1 yields $\phi^{\prime}(r)=0$. Recall that in this case we have $\phi(r)=\psi(r)$.

\subsection{General $\Omega, \nu=1$}

A maximum principle for $\phi$ in this situation has been already proved by the first author:

Theorem 2.2. Let $\Omega$ be a bounded $\kappa$-convex domain, and let $f$ be non-decreasing. If $u$ is an admissible solution to Problem (1), with convex level sets, the function $\phi$ defined in (2) with $\nu=1$ attains its maximum value at the boundary $\partial \Omega$.

Proof. We refer the reader to Theorem 2.1 of [6].

We note that similar maximum (or minimum) principles for more general fully nonlinear equations have been obtained in [2], [7], [8] and [9]. In what follows, we will show that we can prove a similar maximum principle (with weaker conditions) for the function $\psi$. More precisely, we have:

Theorem 2.3. Let $\Omega$ be a $\kappa$-convex domain, and let $f$ be non-decreasing. If $u$ is a $\kappa$-convex solution to Problem (1), the function $\psi$ defined in (3) with $\nu=1$ attains its maximum value at the boundary $\partial \Omega$.

Proof. With $\nu=1$ the function $\psi$ reads as

$$
\psi(x)=u_{k} u_{k}-2\left(\begin{array}{l}
n \\
\kappa
\end{array}\right)^{-\frac{1}{\kappa}} c_{0}^{\frac{1}{\kappa}} u .
$$


We find

$$
\psi_{i i}=2 u_{i i k} u_{k}+2 u_{k i} u_{k i}-2\left(\begin{array}{c}
n \\
\kappa
\end{array}\right)^{-\frac{1}{\kappa}} c_{0}^{\frac{1}{\kappa}} u_{i i} .
$$

Let us make a suitable rotation around a point $\bar{x}$ such that $D^{2} u$ is diagonal at $\bar{x}$. Then,

$$
\psi_{i i}=2 u_{i i k} u_{k}+2 u_{i i} u_{i i}-2\left(\begin{array}{c}
n \\
\kappa
\end{array}\right)^{-\frac{1}{\kappa}} c_{0}^{\frac{1}{\kappa}} u_{i i}
$$

Since

$$
T_{(\kappa-1)}^{i i} u_{i i}=\kappa \sigma_{(\kappa)}=\kappa f
$$

we get

$$
T_{(\kappa-1)}^{i i} \psi_{i i}=2\left[T_{(\kappa-1)}^{i i} u_{i i k} u_{k}+T_{(\kappa-1)}^{i i} u_{i i} u_{i i}-\left(\begin{array}{c}
n \\
\kappa
\end{array}\right)^{-\frac{1}{\kappa}} c_{0}^{\frac{1}{\kappa}} \kappa f\right] .
$$

From Equation (1) we find

$$
T_{(\kappa-1)}^{i j} u_{i j k}=f^{\prime} u_{k}
$$

Since $D^{2} u$ is supposed to be diagonal at $\bar{x}$, then $T_{(\kappa-1)}$ is also diagonal, so

$$
T_{(\kappa-1)}^{i i} u_{i i k} u_{k}=f^{\prime} u_{k} u_{k}
$$

We claim that

$$
T_{(\kappa-1)}^{i i} u_{i i} u_{i i} \geq\left(\begin{array}{l}
n \\
\kappa
\end{array}\right)^{-\frac{1}{\kappa}} f^{\frac{1}{\kappa}} \kappa f .
$$

Indeed, we know that

$$
T_{(\kappa)}=\sigma_{(\kappa)} I-T_{(\kappa-1)} D^{2} u,
$$

from which we find

$$
T_{(\kappa-1)}\left(D^{2} u\right)^{2}=\sigma_{(\kappa)} D^{2} u-T_{(\kappa)} D^{2} u .
$$

Therefore,

$$
T_{(\kappa-1)}^{i i} u_{i i} u_{i i}=\operatorname{trace}\left(T_{(\kappa-1)}\left(D^{2} u\right)^{2}\right)=\sigma_{(\kappa)} \operatorname{trace}\left(D^{2} u\right)-T_{(\kappa)}^{i i} u_{i i},
$$

and

$$
T_{(\kappa-1)}^{i i} u_{i i} u_{i i}=\sigma_{(\kappa)} \sigma_{(1)}-(\kappa+1) \sigma_{(\kappa+1)} .
$$

In view of the latter equation, (14) holds if

$$
\sigma_{(\kappa)} \sigma_{(1)} \geq(\kappa+1) \sigma_{(\kappa+1)}+\left(\begin{array}{c}
n \\
\kappa
\end{array}\right)^{-\frac{1}{\kappa}} \sigma_{(\kappa)}^{\frac{1}{\kappa}} \kappa \sigma_{(\kappa)} .
$$

We know that

$$
\sigma_{(1)} \geq n\left(\begin{array}{l}
n \\
\kappa
\end{array}\right)^{-\frac{1}{\kappa}} \sigma_{(\kappa)}^{\frac{1}{\kappa}}
$$




$$
\sigma_{(1)} \geq n\left(\begin{array}{c}
n \\
\kappa+1
\end{array}\right)^{-\frac{1}{\kappa+1}} \sigma_{(\kappa+1)}^{\frac{1}{\kappa+1}}
$$

and

$$
\sigma_{(\kappa)} \geq\left(\begin{array}{c}
n \\
\kappa
\end{array}\right)\left(\begin{array}{c}
n \\
\kappa+1
\end{array}\right)^{-\frac{\kappa}{\kappa+1}} \sigma_{(\kappa+1)}^{\frac{\kappa}{\kappa+1}}
$$

Now, by (16) we find

$$
\frac{\kappa}{n} \sigma_{(\kappa)} \sigma_{(1)} \geq\left(\begin{array}{l}
n \\
\kappa
\end{array}\right)^{-\frac{1}{\kappa}} \sigma_{(\kappa)}^{\frac{1}{\kappa}} \kappa \sigma_{(\kappa)} .
$$

By (17) and (18) we find

$$
\left(1-\frac{\kappa}{n}\right) \sigma_{(\kappa)} \sigma_{(1)} \geq(\kappa+1) \sigma_{(\kappa+1)} \text {. }
$$

Adding (19) and (20) we find (15), and (14) is proved.

Finally, insertion of (13) and (14) into (12) yields

$$
T_{(\kappa-1)}^{i i} \psi_{i i} \geq 2\left[f^{\prime} u_{k} u_{k}+\left(\begin{array}{c}
n \\
\kappa
\end{array}\right)^{-\frac{1}{\kappa}}\left(f^{\frac{1}{\kappa}}-c_{0}^{\frac{1}{\kappa}}\right) \kappa f\right] \geq 0 .
$$

Since $u$ is $\kappa$-convex, the Newton matrix $T_{(\kappa-1)}$ is positive definite. Therefore, the theorem follows from the classical maximum principle applied to the latter inequality.

\subsection{General $\Omega, \nu=n$}

A maximum principle for $\psi$ (defined in (3)), when $\kappa=\nu=n$, has been proved by Feng and Shi (see [11], Theorem 1.1). When $f=1$, the last result was proved in [4]. We discuss here the case of the P-function $\phi$ (defined in (2)). When $\kappa=\nu=n=2$, a maximum principle has been obtained by the first and the third authors in [10]. In what follows we obtain an extension of their result to any higher dimension:

Theorem 2.4. Let $\Omega$ be a convex domain, and let $f$ be non-decreasing. If $u$ is a convex solution to Problem (1) with $\kappa=n$, the function $\phi$ defined in (2) with $\nu=n$ attains its maximum value at the boundary $\partial \Omega$.

Proof. Our method is very similar to that used in $[4,11]$. Since $T_{(n)}=O$ (the null matrix) and $\sigma_{(n)}=f$, from the equation

$$
T_{(n)}=\sigma_{(n)} I-T_{(n-1)} D^{2} u,
$$

we find

$$
T_{(n-1)}=\left(D^{2} u\right)^{-1} f .
$$

If $\left[u^{k l}\right]=\left(D^{2} u\right)^{-1}$, the P-function (2) with $\nu=n$ reads as

$$
\phi(x):=u^{k l} u_{k} u_{l} f-2 \int_{0}^{u} f(s) d s .
$$


We compute

$$
\phi_{i}=u_{i}^{k l} u_{k} u_{l} f+2 u^{k l} u_{k i} u_{l} f+u^{k l} u_{k} u_{l} u_{i} f^{\prime}-2 f u_{i}
$$

Since

$$
u^{k l} u_{k i}=\delta_{i}^{l} \quad \text { (the Kroneckel delta function) }
$$

we find

$$
\phi_{i}=u_{i}^{k l} u_{k} u_{l} f+u^{k l} u_{k} u_{l} u_{i} f^{\prime}, \quad i=1, \cdots, n .
$$

Further differentiation yields

$$
\begin{aligned}
\phi_{i i}= & u_{i i}^{k l} u_{k} u_{l} f+2 u_{i}^{k l} u_{k i} u_{l} f+2 u_{i}^{k l} u_{k} u_{l} u_{i} f^{\prime} \\
& +2 u^{k l} u_{k i} u_{l} u_{i} f^{\prime}+u^{k l} u_{k} u_{l} u_{i i} f^{\prime}+u^{k l} u_{k} u_{l} u_{i}^{2} f^{\prime \prime} .
\end{aligned}
$$

Next, we make a suitable rotation around a point $\bar{x}$ such that $D^{2} u$ is diagonal at $\bar{x}$. In particular, for $i$ fixed we have

$$
u^{i i} u_{i i}=1, \quad i=1, \cdots, n .
$$

Then we find

$$
\begin{aligned}
\phi_{i i}= & u_{i i}^{k l} u_{k} u_{l} f+2 u_{i}^{i l} u_{i i} u_{l} f+2 u_{i}^{k l} u_{k} u_{l} u_{i} f^{\prime} \\
& +2 u_{i}^{2} f^{\prime}+u^{l l} u_{l}^{2} u_{i i} f^{\prime}+u^{l l} u_{l}^{2} u_{i}^{2} f^{\prime \prime}, \quad i=1, \cdots, n .
\end{aligned}
$$

Multiplying by $u^{i i}$ (and adding from $i=1$ up to $i=n$ ) we get

$$
\begin{aligned}
u^{i i} \phi_{i i}= & u^{i i} u_{i i}^{k l} u_{k} u_{l} f+2 u_{i}^{i l} u_{l} f+2 u^{i i} u_{i}^{k l} u_{k} u_{l} u_{i} f^{\prime} \\
& +2 u^{i i} u_{i}^{2} f^{\prime}+n u^{l l} u_{l}^{2} f^{\prime}+u^{i i} u^{l l} u_{l}^{2} u_{i}^{2} f^{\prime \prime} .
\end{aligned}
$$

Since $\left(D^{2} u\right)^{-1}=T_{n-1}$ is divergence free (see [15]) we have

$$
u_{i}^{i l}=0, \quad l=1, \cdots, n .
$$

Therefore,

$$
\begin{aligned}
u^{i i} \phi_{i i} & =u^{i i} u_{i i}^{k l} u_{k} u_{l} f+2 u^{i i} u_{i}^{k l} u_{k} u_{l} u_{i} f^{\prime} \\
& +2 u^{i i} u_{i}^{2} f^{\prime}+n u^{l l} u_{l}^{2} f^{\prime}+u^{i i} u^{l l} u_{l}^{2} u_{i}^{2} f^{\prime \prime} .
\end{aligned}
$$

Differentiating equation (1) (with $\kappa=n$ ) with respect to $x^{k}$ we find

$$
\frac{\partial \sigma_{(n)}}{\partial u_{i j}} u_{i j k}=f^{\prime} u_{k}, \quad k=1, \cdots, n .
$$

Recalling that

$$
\frac{\partial \sigma_{(n)}}{\partial u_{i j}}=T_{(n-1)}^{i j}=f u^{i j}
$$

we have

$$
f u^{i j} u_{i j k}=f^{\prime} u_{k}
$$


and

$$
u^{i j} u_{i j k}=\frac{f^{\prime} u_{k}}{f} .
$$

Further differentiation yields,

$$
u^{i j} u_{i j k l}+u_{l}^{i j} u_{i j k}=\frac{f\left(f^{\prime \prime} u_{k} u_{l}+f^{\prime} u_{k l}\right)-\left(f^{\prime}\right)^{2} u_{k} u_{l}}{f^{2}}
$$

Since

$$
u^{i l} u_{l j}=\delta_{j}^{i}, \quad i, j=1, \cdots, n,
$$

we have

$$
u_{l}^{i j}=-u^{i p} u^{j q} u_{p q l}
$$

At the point $\bar{x}$ we have

$$
u_{l}^{i j}=-u^{i i} u^{j j} u_{i j l} .
$$

By (24) and the latter equation, at $\bar{x}$ we find

$$
u^{i i} u_{i i k l}=u^{i i} u^{j j} u_{i j l} u_{i j k}+\frac{f\left(f^{\prime \prime} u_{k} u_{l}+f^{\prime} u_{k l}\right)-\left(f^{\prime}\right)^{2} u_{k} u_{l}}{f^{2}} .
$$

Let us now write (25) as

$$
u_{i}^{k l}=-u^{k p} u^{l q} u_{p q i}
$$

Further differentiation yields, for $i=1, \cdots, n$,

$$
\begin{aligned}
u_{i i}^{k l} & =\left(u^{k s} u^{r q} u^{p l}+u^{k q} u^{p s} u^{r l}\right) u_{p q i} u_{r s i}-u^{k p} u^{l q} u_{p q i i} \\
& =2 u^{k k} u^{l l} u^{j j} u_{j k i} u_{j l i}-u^{k k} u^{l l} u_{k l i i} .
\end{aligned}
$$

Multiplying by $u^{i i}$ we find

$$
u^{i i} u_{i i}^{k l}=2 u^{i i} u^{j j} u^{k k} u^{l l} u_{j k i} u_{j l i}-u^{k k} u^{l l} u^{i i} u_{i i k l} .
$$

Insertion of (26) into (27) leads to

$$
\begin{aligned}
u^{i i} u_{i i}^{k l} & =2 u^{i i} u^{j j} u^{k k} u^{l l} u_{j k i} u_{j l i} \\
& -u^{k k} u^{l l}\left(u^{i i} u^{j j} u_{i j l} u_{i j k}+\frac{f\left(f^{\prime \prime} u_{k} u_{l}+f^{\prime} u_{k l}\right)-\left(f^{\prime}\right)^{2} u_{k} u_{l}}{f^{2}}\right) .
\end{aligned}
$$

Simplifying we get

$$
u^{i i} u_{i i}^{k l}=u^{i i} u^{j j} u^{k k} u^{l l} u_{j k i} u_{j l i}-u^{k k} u^{l l} \frac{f\left(f^{\prime \prime} u_{k} u_{l}+f^{\prime} u_{k l}\right)-\left(f^{\prime}\right)^{2} u_{k} u_{l}}{f^{2}} .
$$

Therefore, since (for $l$ fixed) $u^{l l} u_{l l}=1$, we have

$$
\begin{aligned}
u^{i i} u_{i i}^{k l} u_{k} u_{l} f & =u^{i i} u^{j j}\left(u^{k k} u_{j k i} u_{k}\right)^{2} f-\left(u^{k k} u_{k}^{2}\right)^{2} f^{\prime \prime} \\
& -u^{k k} u_{k}^{2} f^{\prime}+u^{k k} u^{l l} \frac{\left(f^{\prime}\right)^{2}}{f} u_{k}^{2} u_{l}^{2} .
\end{aligned}
$$


Inserting (25) and (29) into (22) we find

$$
\begin{aligned}
u^{i i} \phi_{i i} & =u^{i i} u^{j j}\left(u^{k k} u_{j k i} u_{k}\right)^{2} f-\left(u^{k k} u_{k}^{2}\right)^{2} f^{\prime \prime}+u^{k k} u^{l l} \frac{\left(f^{\prime}\right)^{2}}{f} u_{k}^{2} u_{l}^{2} \\
& +(n+1) u^{k k} u_{k}^{2} f^{\prime}-2 u^{i i} u^{k k} u^{l l} u_{k l i} u_{k} u_{l} u_{i} f^{\prime}+\left(u^{i i} u_{i}^{2}\right)^{2} f^{\prime \prime} .
\end{aligned}
$$

Hence,

$$
u^{i i} \phi_{i i}=u^{i i} u^{j j}\left(u^{k k} u_{j k i} u_{k}-\frac{f^{\prime}}{f} u_{i} u_{j}\right)^{2} f+(n+1) u^{k k} u_{k}^{2} f^{\prime} \geq 0 .
$$

Since $u$ is convex, the matrix $\left(D^{2} u\right)^{-1}$ is positive definite. Therefore, the theorem follows by (30) and the classical maximum principle.

Remarks. (i) Consider a $n \times n$ symmetric positive definite matrix $A$ such that

$$
\operatorname{det} A=1
$$

If $v(x)=u(A x)$ then

$$
\begin{aligned}
& D v=A D u, \\
& D^{2} v=A D^{2} u A, \\
& \left(D^{2} v\right)^{-1}=A^{-1}\left(D^{2} u\right)^{-1} A^{-1} .
\end{aligned}
$$

Hence,

$$
\operatorname{det} D^{2} v=\operatorname{det} D^{2} u
$$

and

$$
\begin{aligned}
v^{i j} v_{i} v_{j} & =(D v)^{t}\left(D^{2} v\right)^{-1} D v=(D u)^{t} A A^{-1}\left(D^{2} u\right)^{-1} A^{-1} A D u \\
& =(D u)^{t}\left(D^{2} u\right)^{-1} D u=u^{i j} u_{i} u_{j} .
\end{aligned}
$$

It follows that equation (1) and the P-function (2) with $\nu=n$ are invariant under the transformation $x \rightarrow A x$. Clearly this transformation maps the domain $\Omega$ into $A(\Omega)$, but it preserves the volume, since $\operatorname{det} A=1$.

(ii) As far as we know, there is no maximum principle known for $\phi$ or $\psi$, when $2<\nu<n$, except the one for the case of a ball, obtained in this paper. Therefore, the problem of finding a similar maximum principle for general domains remain open.

\section{Overdetermined problems}

Let us first recall a result proved by the first author:

Theorem 3.1. Let $\Omega$ be strictly convex, and let $u$ be an admissible solution of the problem (1) with $f=\left(\begin{array}{l}n \\ \kappa\end{array}\right)$. If u satisfies the following further boundary condition

$$
H_{(\kappa-1)}|D u|^{\kappa-1}=\left(\begin{array}{c}
n-1 \\
\kappa-1
\end{array}\right) \text { on } \partial \Omega,
$$

then $\Omega$ must be a ball. 
Proof. We refer the reader to Theorem 3.2 (i) of [6].

We prove here the following generalization.

Theorem 3.2. Let $\Omega$ be strictly convex, and let $u$ be an admissible solution of the problem (1), with $f=\left(\begin{array}{l}n \\ \kappa\end{array}\right)$. Fix an integer $r$ such that $1 \leq r<\kappa$. If u satisfies the following further boundary condition

$$
\frac{H_{(\kappa-1)}}{H_{(r-1)}}|D u|^{\kappa-r}=\left(\begin{array}{c}
n-r \\
\kappa-r
\end{array}\right)\left(\begin{array}{c}
\kappa-1 \\
r-1
\end{array}\right)^{-1} \text { on } \partial \Omega,
$$

then $\Omega$ must be a ball.

Proof. Recall the following Newton inequality: if $\lambda_{1}, \cdots, \lambda_{n}$ are the eigenvalues of $D^{2} u$, then we have

$$
\left(\frac{\sigma_{(r)}}{\left(\begin{array}{l}
n \\
r
\end{array}\right)}\right) \geq\left(\frac{\sigma_{(\kappa)}}{\left(\begin{array}{l}
n \\
\kappa
\end{array}\right)}\right)^{\frac{r}{\kappa}}
$$

with equality if and only if $\lambda_{1}=\cdots=\lambda_{n}$ (see [12]).

From equation (1), with $f=\left(\begin{array}{l}n \\ k\end{array}\right)$, we have

$$
\frac{\sigma_{(\kappa)}}{\left(\begin{array}{c}
n \\
\kappa
\end{array}\right)}=1 .
$$

Therefore, by (32) we have

$$
\frac{\sigma_{(r)}}{\left(\begin{array}{c}
n \\
r
\end{array}\right)} \geq \frac{\sigma_{(\kappa)}}{\left(\begin{array}{c}
n \\
\kappa
\end{array}\right)}
$$

Recalling that $T_{(r-1)}$ is divergence free and using Green's formula we find

$$
\begin{aligned}
& \int_{\Omega} \frac{\sigma_{(r)}}{\left(\begin{array}{l}
n \\
r
\end{array}\right)} d x=\left(\begin{array}{l}
n \\
r
\end{array}\right)^{-1} \frac{1}{r} \int_{\Omega} T_{(r-1)}^{i j} u_{i j} d x=\left(\begin{array}{c}
n \\
r
\end{array}\right)^{-1} \frac{1}{r} \int_{\Omega}\left(T_{(r-1)}^{i j} u_{i}\right)_{j} d x \\
& =\left(\begin{array}{l}
n \\
r
\end{array}\right)^{-1} \frac{1}{r} \int_{\partial \Omega} T_{(r-1)}^{i j} u_{i} u_{j} \frac{1}{|D u|} d s=\left(\begin{array}{c}
n \\
r
\end{array}\right)^{-1} \frac{1}{r} \int_{\partial \Omega} H_{(r-1)}|D u|^{r} d s,
\end{aligned}
$$

where the equation

$$
T_{(r-1)}^{i j} u_{i} u_{j}=H_{(r-1)}|D u|^{r+1} \text { on } \partial \Omega
$$

has been used (we refer the reader to [15] for a proof). Similarly, we find

$$
\begin{aligned}
& \int_{\Omega} \frac{\sigma_{(\kappa)}}{\left(\begin{array}{l}
n \\
\kappa
\end{array}\right)} d x=\left(\begin{array}{c}
n \\
\kappa
\end{array}\right)^{-1} \frac{1}{\kappa} \int_{\Omega} T_{(\kappa-1)}^{i j} u_{i j} d x=\left(\begin{array}{c}
n \\
\kappa
\end{array}\right)^{-1} \frac{1}{\kappa} \int_{\Omega}\left(T_{(\kappa-1)}^{i j} u_{i}\right)_{j} d x \\
& =\left(\begin{array}{l}
n \\
\kappa
\end{array}\right)^{-1} \frac{1}{\kappa} \int_{\partial \Omega} T_{(\kappa-1)}^{i j} u_{i} u_{j} \frac{1}{|D u|} d s=\left(\begin{array}{c}
n \\
\kappa
\end{array}\right)^{-1} \frac{1}{\kappa} \int_{\partial \Omega} H_{(\kappa-1)}|D u|^{\kappa} d s .
\end{aligned}
$$

Using the boundary condition (31) we have

$$
\left(\begin{array}{c}
n \\
r
\end{array}\right)^{-1} \frac{1}{r} \int_{\partial \Omega} H_{(r-1)}|D u|^{r} d s=\left(\begin{array}{c}
n \\
\kappa
\end{array}\right)^{-1} \frac{1}{\kappa} \int_{\partial \Omega} H_{(\kappa-1)}|D u|^{\kappa} d s .
$$


Therefore, from (34) and (35) we find

$$
\int_{\Omega}\left(\frac{\sigma_{(r)}}{\left(\begin{array}{l}
n \\
r
\end{array}\right)}-\frac{\sigma_{(\kappa)}}{\left(\begin{array}{l}
n \\
\kappa
\end{array}\right)}\right) d x=0 .
$$

The latter result and inequality (33) imply

$$
\frac{\sigma_{(r)}}{\left(\begin{array}{l}
n \\
r
\end{array}\right)}=\frac{\sigma_{(\kappa)}}{\left(\begin{array}{l}
n \\
\kappa
\end{array}\right)}, \quad x \in \Omega
$$

Since

$$
\frac{\sigma_{(\kappa)}}{\left(\begin{array}{l}
n \\
\kappa
\end{array}\right)}=1
$$

we can also write our previous equation as

$$
\frac{\sigma_{(r)}}{\left(\begin{array}{l}
n \\
r
\end{array}\right)}=\left(\frac{\sigma_{(\kappa)}}{\left(\begin{array}{l}
n \\
\kappa
\end{array}\right)}\right)^{\frac{r}{\kappa}}
$$

Then, equality must hold in (32), and the eigenvalues $\lambda_{1}, \cdots, \lambda_{n}$ of $D^{2} u$ must be equal. By Equation (1) with $f=\left(\begin{array}{l}n \\ \kappa\end{array}\right), D^{2} u$ is the identity matrix, and the result follows.

We consider now an overdetermined problem already solved in [3] by using a different method. We need the following Pohozaev type identity.

Lemma 3.3. If $\Omega$ is $\kappa$-convex, if $e=\left(e^{1}, \cdots, e^{n}\right)$ is the exterior normal to $\partial \Omega$ and if $u$ is an admissible solution to problem (1) with $f=1$, then

$$
\int_{\partial \Omega} x^{\ell} e^{\ell} T_{(\kappa-1)}^{i j} u_{i} u_{j} d s=\kappa(n+2) \int_{\Omega}(-u) d x .
$$

Proof. Since $u=0$ on $\partial \Omega$ we have $u_{j}=|D u| e^{j}$ and $e^{\ell}=\frac{u_{\ell}}{|D u|}$. Therefore,

$$
\begin{aligned}
& \int_{\partial \Omega} x^{\ell} e^{\ell} T_{(\kappa-1)}^{i j} u_{i} u_{j} d s=\int_{\partial \Omega} x^{\ell} u_{\ell} T_{(\kappa-1)}^{i j} u_{i} e^{j} d s=\int_{\Omega}\left(x^{\ell} u_{\ell} T_{(\kappa-1)}^{i j} u_{i}\right)_{j} d x \\
& =\int_{\Omega} T_{(\kappa-1)}^{i j} u_{i} u_{j} d x+\int_{\Omega} x^{\ell} u_{\ell j} T_{(\kappa-1)}^{i j} u_{i} d x+\int_{\Omega} x^{\ell} u_{\ell} T_{(\kappa-1)}^{i j} u_{i j} d x .
\end{aligned}
$$

Integrating by parts and using the fact that $T_{(\kappa-1)}$ is divergence free, we find

$$
\int_{\Omega} T_{(\kappa-1)}^{i j} u_{i} u_{j} d x=\int_{\Omega} T_{(\kappa-1)}^{i j} u_{i j}(-u) d x=\kappa \int_{\Omega}(-u) d x .
$$

Next, since $\sigma_{(\kappa)}=1$ we have

$$
T_{(\kappa)}=I-T_{(\kappa-1)} D^{2} u,
$$

and

$$
T_{(\kappa-1)}^{i j} u_{\ell j}=\delta_{\ell}^{i}-T_{(\kappa)}^{i \ell}
$$


On the other hand, since $I-T_{(\kappa)}$ is divergence free, it follows that

$$
\left(u_{\ell j} T_{(\kappa-1)}^{i j}\right)_{i}=0
$$

Therefore,

$$
\begin{aligned}
& \int_{\Omega} x^{\ell} u_{\ell j} T_{(\kappa-1)}^{i j} u_{i} d x=\int_{\Omega}\left(x^{\ell} u_{\ell j} T_{(\kappa-1)}^{i j}\right)_{i}(-u) d x \\
& =\int_{\Omega} \delta_{i}^{\ell} u_{\ell j} T_{(\kappa-1)}^{i j}(-u) d x=\kappa \int_{\Omega}(-u) d x .
\end{aligned}
$$

Finally, we also have

$$
\int_{\Omega} x^{\ell} u_{\ell} T_{(\kappa-1)}^{i j} u_{i j} d x=\kappa \int_{\Omega} x^{\ell} u_{\ell} d x=\kappa n \int_{\Omega}(-u) d x .
$$

Inserting now (38), (39) and (40) into (37), we are lead to (36). The proof of the lemma is thus achieved.

Now let us consider problem (1), with $\kappa=n$ and $f=1$. Our new further boundary condition is the following

$$
T_{(n-1)}^{i j} u_{i} u_{j}=c^{2} \text { on } \partial \Omega .
$$

Condition (41) can be written as

$$
H_{(n-1)}|D u|^{n+1}=c^{2} \text { on } \partial \Omega .
$$

We note that the exponent of $|D u|$ is now $n+1$, whereas in Theorem 3.1, with $\kappa=n$, the exponent of $|D u|$ is $n-1$. Condition (41) does not imply that $\Omega$ is a ball. Indeed, condition (41) and equation (1), with $\kappa=n$, are invariant under the linear transformation $y=A x$, where $A$ is a symmetric matrix with $\operatorname{det} A=1$. By Theorem 4.2 of [5], condition (41) appears naturally while optimizing the domain dependent functional

$$
E(\Omega)=\int_{\Omega}\left(T_{(n-1)}^{i j} u_{i} u_{j} u+\left(n+n^{2}\right) u\right) d x
$$

(where $u$ is the solution to Problem (1) with $\kappa=n$ and $f=1$ ), among all domains $\Omega$ of given measure. Note that we can express the previous functional as

$$
E(\Omega)=n^{2} \int_{\Omega} u d x
$$

By Theorem 1.1 of [4] (or our Theorem 2.4 with $f=1$ ), we have

$$
T_{(n-1)}^{i j} u_{i} u_{j}-2 u \leq \max _{\partial \Omega} T_{(n-1)}^{i j} u_{i} u_{j} \quad \forall x \in \Omega .
$$

Theorem 3.4. Let $u$ be a convex solution to Problem (1), with $\kappa=n$ and $f=1$. If we have

$$
T_{(n-1)}^{i j} u_{i} u_{j}=c^{2} \quad \text { on } \partial \Omega
$$

then equality holds in (42). 
Proof. By Lemma 3.3, with $\kappa=n$, we have

$$
\int_{\partial \Omega} x^{\ell} e^{\ell} T_{(n-1)}^{i j} u_{i} u_{j} d s=n(n+2) \int_{\Omega}(-u) d x .
$$

Using condition (43), from the latter equation we find

$$
c^{2} \int_{\partial \Omega} x^{\ell} e^{\ell} d \sigma=n(n+2) \int_{\Omega}(-u) d x
$$

so

$$
c^{2}|\Omega|=(n+2) \int_{\Omega}(-u) d x
$$

On the other hand, integration of (42) over $\Omega$ yields

$$
\int_{\Omega} T_{(n-1)}^{i j} u_{i} u_{j} d x+2 \int_{\Omega}(-u) d x \leq c^{2}|\Omega| \text {. }
$$

Since

$$
\int_{\Omega} T_{(n-1)}^{i j} u_{i} u_{j} d x=\int_{\Omega} T_{(n-1)}^{i j} u_{i j}(-u) d x=n \int_{\Omega}(-u) d x
$$

from (45) we find

$$
(n+2) \int_{\Omega}(-u) d x \leq c^{2}|\Omega| .
$$

By (44), equality holds in (46). Hence, equality must hold in (45), and the assertion of the theorem follows. The proof is thus achieved.

Remarks. (i) Under the conditions of Theorem 3.4, we find $c^{2}=-2 u_{\min }$. Hence,

$$
T_{(n-1)}^{i j} u_{i} u_{j}=2\left(u-u_{\text {min }}\right), \quad x \in \Omega .
$$

As a consequence, if $u_{\text {min }}<t<0$ and if $\Omega_{t}=\{x \in \Omega: u(x)<t\}$, we have

$$
T_{(n-1)}^{i j} u_{i} u_{j}=2\left(t-u_{\min }\right) \quad \text { on } \partial \Omega_{t}
$$

so

$$
H_{(n-1)}\left(\partial \Omega_{t}\right)|D u|^{n+1}=2\left(t-u_{m i n}\right) \quad \text { on } \partial \Omega_{t}
$$

(ii) A solution of the overdetermined problem of Theorem 3.4 is an ellipse if $n=2$ (see [1]) and an ellipsoid if $n>2$ (see [3]).

\section{Acknowledgement}

The first author was supported by an FRG grant from the American University of Sharjah, project number FRG20-S-S117. The second author is a member of the Gruppo Nazionale per l'Analisi Matematica, la Probabilitá e le loro Applicazioni (GNAMPA) of the Istituto Nazionale di Alta Matematica (INdAM). Furthermore, she is partially supported by the research project: Evolutive and stationary Partial Differential Equations with a focus on biomathematics (Fondazione di Sardegna 2019). 


\section{Bibliography}

[1] C. Anedda, G. Porru, Problems on the Monge-Ampère equation in the plane, Contemporary Mathematics, Volume 400 (2006), 1-9.

[2] L. Barbu, C. Enache, A maximum principle for some fully nonlinear elliptic equations with applications to Weingarten surfaces, Complex Var. Elliptic Equ. 58 (2013), 1725-1736.

[3] B. Brandolini, N. Gavitone, C. Nitsch, C. Trombetti, Characterization of ellipsoids through an overdetermined boundary value problem of Monge-Ampère type, Journal de Mathématiques Pures et Appliquées, 101, (2014), 828-841.

[4] C. Chen, X. Ma, S. Shi, Curvature estimates for the level sets of solutions to the Monge-Ampère equation $D^{2} u=1$, Chin. Ann. Math., Series B (2014), 895-906.

[5] B. Emamizadeh, Y. Liu and G. Porru (2020): Overdetermined problems for p-Laplace and generalized Monge-Ampère equations, Complex Variables and Elliptic Equations, DOI: 10.1080/17476933.2020.1843447

[6] C. Enache, Maximum principles and symmetry results for a class of fully nonlinear elliptic PDEs, NoDEA Nonlinear Differ. Equ. Appl. 17 (5) (2010) 591-600.

[7] C. Enache, Maximum and minimum principles for a class of Monge-Ampère equations in the plane, with applications to surfaces of constant Gauss curvature, Commun. Pure Appl. Anal. 13 (2014) 1347-1359.

[8] C. Enache, Maximum principles and isoperimetric inequalities for some Monge-Ampère type problems, C. R. Math. Acad. Sci. Paris 352(1) (2014) 37--42.

[9] C. Enache, Necessary conditions of solvability and isoperimetric estimates for some Monge-Ampère problems in the plane, Proc. Amer. Math. Soc. 143 (2015) 309-315.

[10] C. Enache, G. Porru, A Note on Monge-Ampère Equation in $\mathbb{R}^{2}$, Results Math. 76(1) (2021) 1-8.

[11] H. Feng, S. Shi, Curvature estimates for the level sets of solutions to a class of Monge-Ampère equations, Nonlinear Analysis 178 (2019), 337-347.

[12] G.H. Hardy, J.E. Littlewood and G. Pólya, Inequalities, Cambridge Mathematical Library. Cambridge University Press, Cambridge (1998).

[13] L.E. Payne, G.A. Philippin, On maximum principles for a class of nonlinear second-order elliptic equations. J. Differential Equations 37(1) (1980), 39-48.

[14] M.H. Protter, H.F. Weinberger, Maximum principles in differential equations, Springer (1999).

[15] R.C. Reilly, On the Hessian of a function and the curvatures of its graph, Michigan Math. J. 20 (1973), 373-383.

[16] P. Salani, Boundary blow-up problems for Hessian equations, Manuscripta Math. 96 (1998), 281-294.

[17] J. Serrin, A symmetry problem in potential theory, Arch. Rat. Mech. Anal. 43 (1971), 304-318.

[18] R.P. Sperb, Maximum Principles and Their Applications, Mathematics in Science and Engineering, vol. 157, Academic Press, New York, 1981, ix+224 p.

[19] H. Weinberger, Remark on the preceding paper by Serrin, Arch. Rat. Mech. Anal. 43 (1971), 319-320. 